\title{
L'ordine del discorso. Gli influssi della cultura francese contemporanea in Italia.
}

Qualche tempo fa gli ambienti letterari italiani furono messi in subbuglio da un'ennesima querelle. ${ }^{1}$ Che era accaduto? Alcuni critici erano giunti nella convinzione che strutturalismo e semiologie, in tutte le loro varianti e declinazioni, fossero da buttare una volta per sempre. Si trattava di un'impennata di studiosi che si muovevano in un'area in cui tra letteratura e ideologia correvano rapporti abbastanza stretti; tanto che non stupisce che si fossero determinati a partire a oste contro un avversario sprezzante e sussiegoso, pieno di una conclamata scientificità dei propri strumenti ma spesso esulante dai conti del testo, voglio dire dalle sue componenti variabili, non racchiuse in nessun grafico, quadrante o triangolino.

Ciò che soprattutto destò meraviglia fu che i molti colpi assestati a quella botte dai molti cerchi - ai blasonati della scienza letteraria - non incontrassero sostanzialmente reazioni: e questo è il vero punto. La liquidazione di quindici anni e piú di analisi metodologie griglie schidionature paradigmi ecc. ecc., si appuntò lontanissima da ogni contestazione. Qualcuno - vedi Cesare Segre in Tutto libri ${ }^{2}$ - trovò modo di osservare che in ogni caso lo strutturalismo, in un panorama accidentato e approssimativo come quello italiano, era servito a qualcosa, se non altro a ripulire dal vecchiume. E tuttavia un "de profundis" cosí reciso e anche ingeneroso mai fu recitato con un consenso, o un'indifferenza, pressoché generali, soprattutto da parte dei molti che, ancora in un passato recente, avevano consuonato nel loro lavoro con le tendenze e le pratiche adesso accantonate. 
Di quale pasta (ma il vocabolario impiegato, come si vedrà, volge molto all'eufemismo) fossero questi voltabandiera, o allegri saltatori delle patrie lettere, è ben detto da Alfonso Berardinelli, che è tra coloro che piú impietosamente hanno scaricato le proprie bordate. Il titolo del suo articolo, apparso nel terzo fascicolo di Linea d'ombra, è "Tipi intellettuali: ruspa, tritacarne, apriscatole, frullatore" e cosí inizia: "L'intellettuale-ruspa sa spianare ampi spazi di terreno davanti a sé. Abbatte senza pietà vecchi edifici traballanti. Le sfumature non sono il suo forte. La sua scrittura e il suo modo di procedere hanno qualcosa di irresistibile, irrefutabile, igienicamente riduttivo e distruttivo: in breve, qualcosa di fragoroso. A un intellettuale-ruspa non si può chiedere di porgere orecchio a ciò che si ode appena. La sua mente è un motore che tutte le mattine si mette in moto per un certo numero di ore e che spiana su un notevole numero di cartelle un notevole numero di temi. La sua specialità è appunto spianare, appianare, appiattire, aprire uno spazio vuoto dove prima si ergeva qualche vecchia costruzione ricca di fascino e di incrostazioni inutili o troppo soggettive." ${ }^{3}$

Nella stessa rivista Franco Brioschi risponde a un intervento abbastanza puntuto di Maria Corti in Alfabeta del luglio-agosto 1983, ${ }^{4}$ rimarcando l'esigenza di una nuova teoria della letteratura - e non già unicamente di uno strumentario analitico - in lega con una coscienza filosofica e culturale, essa sí agguerrita, dei problemi nuovi che ormai si prospettano alla critica. Ma precisa: "la verità è che comunque noi leggiamo un'opera spinti non solo da motivazioni estetiche (ammesso che siano definibili), ma anche e soprattutto da motivazioni extraestetiche: né il cielo della teoria può limitarsi a riflettere le prime (piú o meno arbitrariamente identificate), ignorando le seconde."

Il contenzioso come si vede s'allarga a comprendere tanto il senso quanto la natura dell'oggetto estetico. A un' estremizzazione - quella della forma onnicomprensiva - si risponde insomma con una contrapposta ma in concreto speculare estremizzazione. Molto piú avvedutamente Franco Fortini, in quella sua rubrica nel Corriere della Sera che egli ha intitolato "Questioni di frontiera"6 per figurar il carattere interdisciplinare del dover essere di ogni ricerca letteraria e insieme la posizione di distanza di fronte alle idee dominanti, riammaglia il discorso della politica con quello estetico. La scissura che all'incirca cent'anni fa Stéphane Mallarmé aveva creduto di riconoscere tra estetica da un lato, e economia politica dall'altro, appresso le esperienze, le delusioni, i ripensa- 
menti degli ultimi decenni forse avrebbe potuto sciogliersi in una sorta di scambio, replicato dal rapporto tra politica ed etica.

$\grave{E}$, questo di Fortini, un ragionamento puntuale e stimolante, suscettibile in quel suo modo di aprire un gioco d'echi tra le diverse parti componenti un'indagine critica. Ma non su questo vogliamo richiamare l'attenzione. Ciò che fa punto è il dibattito nel suo complesso, e piú precisamente le ragioni e motivazioni che gli si accorpano dietro. E infatti: come all'affermarsi di strutturalismo e semiologia non furono per nulla estranee le risultanze e le specifiche riflessioni di alcune teste d'uovo della cultura francese (da Claude Lévi-Straus a Barthes, da Todorov a Maurice Blanchot), in eguale misura la loro cancellazione, o come meglio usa dire il loro superamento, ha incontrato una replica presso che meccanica sul versante italiano. È il primo paradosso di una situazione culturale, prima eccettuatasi da stressanti resoconti sociologici, dopo immersa in acque ideologiche (sul finire del decennio '60-'70), infine imbarcatasi beatamente nel delirio esclamativo e pseudolirico degli ultimi anni. A tutti questi passaggi - la definizione acutamente adottata da Fortini è "flessibilità"7 — hanno contribuito le complesse e alterne vicende dell'intellighenzia parigina. Non soltanto quelle, ma anche quelle.

Il nodo da sciogliere, è capire come la Francia appaia oggi agli italiani colti, meglio agli intellettuali di professione. Gli Stati Uniti sono sentiti in modo centrale, come il solo paese che dia una risposta a livello planetario alle esigenze vuoi della tecnica vuoi del simbolico collettivo (nella versione, opinabile e ambigua, dell'immaginario di massa). Ma la Francia, invece, in quel suo continuo elaborare mode culturali e parole à la page, è intesa come la provincia dello strumentario retorico: una sorta di abilità concettuale che consente l'approdo alla realtà corrente. È il laboratorio che fornisce l'indispensabile "trufferia di parole" 8 delle quali dotarsi per solo o almeno mostrare di riconoscere, inseguire, circoscrivere la verità. Piú che mai i nomi e i concetti individuano le cose: nomina sunt res. Ma poi, in concreto, tale verità, contornata e marcata con tutte le sfumature e le risorse della "réthorique," viene pari pari assimilata ai modelli e alle stesse revulsioni dell'American way of life (e estensivamente della sua ideologia unidimensionale). E qui si tocca con mano la seconda palese contraddizione, del resto non celata. Le armi sottili della Francia eurocentrica servono a corroborare il primato statunitense.

Non è, tutto questo, soltanto l'effetto della pallida effulgenza della cultura italiana del dopo-contestazione. Qualcosa di volatile, 
di epidermico, di obliquo, c'è naturalmente anche al fondo dell'attuale situazione francese, non sempre libera dalla fucileria di apparenze e barocchismi che le pesano sopra. Da un'eguale enfiagione non s'è in pari luogo sciolta quella cultura italiana, che ha continuato a girare di riporto sul perno dei discrepanti pareri e argomenti che arrivavano d'Oltralpe. Ma non tutto il negativo ha il rincalzo delle mode parigine, come non tutto ciò che arriva da $\mathrm{Pa}-$ rigi porta il contrassegno della fatuità. Molto spesso è vero il contrario.

Si prenda il diffondersi dello strutturalismo negli anni '60: che è grosso modo il termine post quem da cui far datare la ripresa di influenza francese sugli intellettuali italiani. Scorrendo infatti una qualsiasi pubblicazione sull'argomento, i nomi francesi occupano la quasi totalità dello spazio. Certo, il punto di partenza può essere una produzione, concettuale o non, linguistica o pragmatica, esterna all'enclave parigina (per esempio, Cassirer). Ma ben presto, dal primo germinare di uno strutturalismo metodologico (nella fattispecie cassireriano), si arriva alla fondazione ontologica dell'analisi strutturalista con Claude Lévi-Strauss, fautore di un'ontologia deprivata di essenza, di essere, e del marxista Louis Althusser. Successivamente viene investito l'ambito della letteratura, con Barthes e Blanchot, e si va in direzione della psicoanalisi con Lacan e Foucault (abbiamo casualmente citato da un libro divulgativo sull'argomento, ${ }^{9}$ uno dei tanti).

Sono tutte presenze, quelle ricordate, che puntualmente si ritrovano nel dibattito italiano (che risalendo a piú di vent'anni fa non ci pare il caso di ricostruire), ma che al negativo dell'imitazione accludono l'indubbia positività di mettere al passo con l'Europa, in virtú dell'inattesa scoperta di ambiti di sapere sino ad allora ignorati (se si fa astrazione dalla scuola dellavolpiana). Tali suggestioni maturano ad esempio nell'area del Gruppo '63 e delle avanguardie figurative. Sempre abbreviativamente, deve essere puntualizzato l'impulso che le nuove correnti di pensiero e d'analisi hanno dato alla critica delle arti, e in particolare della letteratura. L'idea che alla scienza e alle scienze umane si potesse affiancare con un suo rinnovato statuto di oggettività la ricerca letteraria ha parecchio eccitato i nostri studiosi e scrittori e professori d'università. Allargandosi da Charles Mauron con la sua rete di metafore ossessive alle prospezioni psicanalitiche - piú che lacaniane alla Lacan - dalla semantica strutturale greimasiana a Roland Barthes, risalendo addietro ai formalisti russi e rivedendo sotto certi aspetti la lezione desanctisiana, tutte le armature del testo lette- 
rario, dalle meno parventi alle piú insistite e sensibili, son state sottoposte a minuziose e persino pedantissime indagini. Ma ancora una volta i francesi, con i materiali prodotti dove con gli incontri e i simposi (ricorderò per tutti i seminari di Cérisy-la-Salle) dove coi libri collettivi (si veda Théorie d'ensemble, per la Collection "Tel Quel" delle edizioni du Seuil), l'hanno fatta da padroni.

Proprio Barthes ha un ruolo di primissimo piano nel mutare degli orientamenti. Nel '73, con Le plaisir du texte, fatto uscire sempre aux éditions du Seuil, affronta e prospetta il problema dello scarto del godimento dell'arte, e del prodotto estetico in genere, dal puritanesimo ideologico in quei tempi ancora galoppante e onnicomprensivo, oltreché dall'asettica sufficienza di tutte le semiologie. Il godimento del testo è per lui un non-detto, anzi meglio una indicibilità: la possibilità di affidarsi all'inordinata sequenza di schegge, tocchi, barbagli, filamenti, illuminazioni, che costituiscono dentro la scrittura la parte insondabile, il fuoriscienza (come lui stesso ebbe ad osservare) dell'analisi testuale. Lo stesso testo che è scritto deve offrire la prova di desiderare il soggetto che scrive. La scrittura è infatti la prova che esso esiste, e diviene per ciò stesso scienza attiva dei godimenti del linguaggio.

Le implicazioni del discorso avviate da Barthes nel suo aureo libriccino (poco meno di settanta pagine), tuttoché non afferrate in tutta la loro portata al suo apparire, offrivano l'abbrivo a una discussione - e a ripensamenti e viraggi di rotta - che avrebbero finito col sovvertire atteggiamenti, pensieri e pratiche, tanto d'analisi che di scrittura, giusto allo scucirsi degli anni '70. Molto apertamente, scompaginando i termini della questione cosí come ognuno vedeva sul tappeto appunto in quel torno d'anni, il saggista francese contrappone al riverbero delle necessarie nuvole aleggianti sulla pagina (la soggettività, i referenti, le ideologie), il chiaroscuro della sovversione; e al contempo sottrae al dominio incontrastato - il testo, il pre-testo, il con-testo - della semiosi segmenti di scrittura inanalizzabili per lo strutturalismo. La letteratura comincia insomma a sfuggire a quelle griglie - sociologiche tanto quanto formali - che le erano state tessute all'intorno.

La posizione di Barthes era di tanto piú interessante, in quanto concerneva cosí la scrittura creativa (lirica, narrativa, drammaturgica, ecc.) come pure quella saggistica. Ma in piú aveva il rincalzo di operare essa stessa, all'interno del testo in cui si formalizzava, come modello non già di stesura argomentativa ma bensí di scrittura tout court. Anzi, come unico modello di scrittura letteraria che 
la Francia avesse da anni esportato ed imposto da noi. Non è stato ugualmente di Althusser Lacan o della Kristeva, o di quello che a nostro vedere è il piú notevole, Michel Foucault, piú riconosciuti e seguiti in forza delle loro qualità di maîtres-à-penser.

Si può ragionevolmente sostenere che la prosa di Barthes non escluse ovviamente le idee e le intuizioni sulle quali si esempla - abbia nella cultura italiana (ma diremmo anche in quella francese) tenuto il luogo che negli anni ' 60 era stato di competenza degli strutturalisti, e in quelli '50 dell'engagement. La differenza è che essa non ha per nulla configurato una corrente, una tensione e una solidarietà di gruppo, una posizione da manifesto con enunciati perentoriamente sillabati: diffondendosi invece con sottile e intonata precisione piú capillarmente e in profondità. Dieci anni di distanza, e il sostanziale mutamento del quadro di riferimento culturale e creativo, le hanno dato oggettivamente ragione. E hanno dato ragione a Barthes. Ma di tutto ciò, si dirà dopo.

Ciò che invece, nello scorcio del decennio cominciato con l'iperideologizzazione e concluso dall'Autonomia e dal terrorismo, mise dimora sul terreno italiano, fu il pensare - o meglio il nonpensare affatto, che nel caso in questione è la stessa cosa - dei sedicenti "nuovi filosofi."

Dopo la critica come lettura, come scienza, come valutazione: la prima confidata a Barthes ("Seule la lecture aime l'oeuvre, entretient avec elle un rapport de désir. Lire, c'est désirer l'oeuvre, c'est vouloir être l'oeuvre, c'est refuser de doubler l'oeuvre en dehors de toute autre parole que la parole même de l'oeuvre, ${ }^{\prime 10}$ è Critique et vérité); la seconda appannaggio delle diverse scuole storiche e filologiche; la terza legata alle molteplici teorie e ideologie di produzione del linguaggio; dopo l'effetto di esemplarità, quantomeno in termini epistemologici, della "nouvelle critique" e il sorgere ad esempio, sulle ceneri dello storicismo, di un materialismo althusseriano e strutturalista, ${ }^{11}$ di un Marx scienziato della società da opporre al Marx umanistico e giovanile (con recuperi quantomeno desueti, come l'Engels non deterministico di Eleonora Fiorani) ${ }^{12}$ dopo insomma il febbrile coniugarsi di innovazione e ideologia, di indagine condotta sulla microstruttura in omologia al piú generale criterio d'indagine dell'ambito sociale e politico della struttura e della sovrastruttura, il proliferare delle energie si indirizzò indistintamente sul nuovo verbo dei Glucksmann e dei Levy. Inutile dire che quel loro biascicare a vuoto colpí come una dissonanza, arrivò come una necessaria dissacrazione. 
Altra certo era la consistenza dei ragionamenti sulla rivoluzione molecolare di Félix Guattari, ${ }^{13}$ o meglio ancora sulla microfisica del potere quale prospettava Foucault ${ }^{14}$ (che trovò un'udienza molto preoccupata ed attenta, ad es. in Alberto Asor Rosa). ${ }^{15} \mathrm{Ma}$ tant'è: pur pretendendo di elevarsi a logos, e intendendo comporsi in un'autonomia categoriale e teoretica, le digressioni di Levy e compagni non giunsero a interessare che in maniera irrilevante il mondo dei pensatori e dei filosofi e invece ingabbiarono ancora una volta $\mathrm{i}$ letterati, le teste d'uovo, i dissidenti di vocazione, gli elzeviristi delle terze pagine dei grandi fogli della borghesia. Pronubi dell'operazione furono gli ambienti della nuova dirigenza socialista; i cosiddetti extraparlamentari; le intelligenze inquiete, si direbbe alla ricerca di una nuova retorica, come facoltà di rilevare quel che in ciascun caso fosse atto a persuadere, oppure a raggiungere indifferentemente essere e non essere.

I "nouveaux philosophes," e con loro i Guattari, i Deleuze, gli Jambet e i Lardreau (un cui volume, L'Angelo, ${ }^{16}$ ontologia della rivoluzione e per una cinegetica del fantasma, appare in italiano con una introduzione di Gianni Scalia), costituirono, insieme con la Heller $^{17}$ (non però appiattibile sulla loro dimensione), un po' il retroterra ideale dell'antirepressione. I loro equivalenti italiani, o i loro vessilliferi ed imitatori - il bolognese Bifo, cimentatosi anche con il romanzo e trasmigrato "sur Seine" dopo i fatti violenti dell'Autonomia a Bologna, il collettivo di Radio Alice, gli indiani metropolitani e i gruppi creativi, i tanti intellettuali che da un impegno ossessivo in ambito politico e dalla critica del revisionismo erano scivolati nel disimpegno piú eclatante (secondo la formula: organizzare il dissenso, disorganizzare il consenso; si pensi alla rivista Il cerchio di gesso) - volsero in niente e, se in qualcosa, in squallore, posizioni per loro conto cosí poco pertinenti e scientifiche come quelle in genere professate dall'eterogeneo e composito gruppuscolo dei nuovi filosofi.

Accadde in breve che per adeguarsi alla nuova mentalità, gli intellettuali italiani - non tutti, certo: ma molti - divennero e perorarono l'opposto di ciò che sino a quel momento essi erano stati. Valga per tutti l'esempio di Leonardo Sciascia che, a malgrado del suo conclamato illuminismo, prefazionò e infarcí di apprezzamenti un libercolo di André Glucksmann (La barbarie del volto umano, 1977), nel quale si sparava a zero - con non contenuta rozzezza e con una ancora piú parvente ignavia teoretica proprio sui fondamenti etici e culturali dell'illuminismo europeo (ad esso opponendo ordures panslavistiche e medievaleggianti, e 
ad esso attribuendo nefasti e fasti del nazismo e dello stalinismo, oltreché della borghesia). Ma, si sa: Parigi vale bene una messa.

Questa del convertirsi al mot juste, o del guidare i propri passi fin dentro il recinto della sacra città e nei salotti che contano, è del resto piú che una propensione una vera e propria strategia di alcuni. Sull'onda del dissenso dagli ex-compagni di strada comunisti e dalle impostazioni del compromesso storico, Leonardo Sciascia approda a Parigi, viatico indispensabile per una ricercata consacrazione internazionale. Un altro deluso dalla politica e dall'ideologia, Italo Calvino, andrà a risciacquare nei torbidi della Senna le sue macchie neorealistiche e vittoriniane: anch'egli tutto sommato - attratto dalla diana della cassa di risonanza francese (che naturalmente, sia per lui che per Sciascia, non mancherà di farsi ben sentire).

Specialmente nel caso dello scrittore siciliano, il nuovo orizzonte della cultura francese, irridente ogni mito e tutte le certezze totalizzanti, è stato il terreno di coltura di alcune sue tematiche. Il giallo morale che aveva contrassegnato le indagini sulle società del nostro meridione, sulla mafia, sul contesto vischioso del potere, metaforizzando nella parallela impotenza un dato esistenziale, prende adesso a slabbrarsi in un racconto piú spicciolo: piú dossier giornalistico da grande rotocalco, che libro inventivo in senso proprio. Con la serie delle inchieste, dei casi e degli enigmi della storia presente ma anche passata, l'arte di Sciascia sembra fare punto. L'avvio è dato, dopo La scomparsa di Majorana (ancora interessante per l'aria di mistero che vi circola), da L'affare Moro, la cui prima edizione è naturalmente in francese: libro il cui fascino è soltanto localizzabile al capitolo introduttivo, nel quale si cita e chiosa il bellissimo articolo di Pier Paolo Pasolini sulla scomparsa delle lucciole. $^{18}$

In via di chiusa è anche riportata una lettera dello statista ucciso dai brigatisti, lettera indirizzata alla moglie Nora e lasciata filtrare, pour cause, dai suoi assassini. Che però per Sciascia, tutto preso nel suo ruolo di indagatore delle verità oltre le facciate ufficiali, sono affidabili e comprensivi e gentili piú di quanti si opposero a che lo Stato trattasse coi terroristi riconoscendo in loro un interlocutore valido, seppure in guerra. Si legga per convincersene il testo integrale, tirato giú da una registrazione, del colloquio telefonico di uno dei terroristi con un professore amico della famiglia Moro, per comunicargli l'avvenuta esecuzione; e si veda poi di seguito l'ineffabile commento sciasciano: "Forse ancora oggi il giovane brigatista crede di credere si possa vivere di odio e contro la 
pietà: ma quel giorno, in quell'adempimento, la pietà è penetrata in lui come il tradimento in una fortezza. E spero che lo devasti."19 Potenza inaudita di una retorica che è salvifica solo a senso unico!

L'Affare Moro esempla infine quella logorrea, dannunziana ed enfatica (si ripensi ai libri di Toni Negri), ${ }^{20}$ che intese pennellare l'Italia dell'epoca come il paese dell'autoritarismo e della repressione, in piena consonanza con ciò che sbavavano gli intellettuali parigini, i vecchi e i nuovi filosofi, i transfughi nelle file della destra conservatrice e antisemita; e in piena consonanza anche con quel loro stile imbastito di citazioni, rombe, eloquio stentoreo e ininterrotto come di chi parli sul crinale tra secolo e secolo, di postille del tutto sprovviste di logica e però affidate al valore del climax e con le figure che prendono campo sulla pagina piú che le idee, senza tuttavia che la scrittura, pezzata da motivi tanto diversi, riammagli un equilibrio, una centralità, una stilematica piú riconoscibile.

Le prodezze dell'intaglio e dei modelli argomentativi, lo stesso appannamento della realtà dei fatti per far rientrare l'indecifrabilità del crimine nelle spire di un labirinto alla Borges, sono i telai che consentono il raffronto con le intelligenze e i gesti esemplari da "grands intellectuels": alla Hugo, alla Zola, alla Sartre. Il tutto ottenuto spostando, deflettendo, ricomponendo il quadro, procedendo sulla via dell'analisi verso un risultato infine ambiguo e illeggibile che lascia al centro la figura carismatica dello scrittore. E tutto in connessione con i deliri, gli asserti, le esplosioni meramente verbali della ultima cultura parigina: ma impostando quel discorso del senso del limite, in cui poi s'innervi la ricerca e l'individuazione della verità.

L'uomo - come volevano gli illuministi - edifica la propria forza su ciò che non può capire. È la lezione di umiltà e autocoscienza del Voltaire di Candide. Sciascia lo trasfonde in quella sua sismografia a raggiera, un "sogno fatto in Sicilia," come dice il sottotitolo del suo Candido svolto sull'ambiguità e le contraddizioni del cattolicesimo fatto confluire nel comunismo e viceversa. Ma precisa: "Dice Montesquieu che 'un'opera originale ne fa quasi sempre nascere cinque o seicento altre, queste servendosi della prima all'incirca come i geometri si servono delle loro formule.' Non so se il Candide sia servito da formula a cinque o seicento altri libri. Credo di no, purtroppo: ché ci saremmo annoiati di meno, su tanta letteratura. Comunque, che questo mio racconto sia il primo o il seicentesimo, di quella formula ho tentato di servirmi. Ma mi pare di non avercela fatta, e che questo libro somigli agli 
altri miei. Quella velocità e leggerezza non è piú possibile ritrovarle: neppure da me." ${ }^{21}$ E infatti l'impresa di tornare su tanto classico, o di servirsi di esso stabilendo un ideale paragone, si risolve in vaniloquio e velleità. Il Candido di Sciascia è un libro iroso e pesante, in cui la miglior prosa del mondo (ricordate il giudizio Borgesiano sul capolavoro di Voltaire?) è disvolta in una non piú che puntuale esercitazione scolastica. Insomma, di Francia si può anche perire.

Ciononpertanto Sciascia resta il solo autore italiano di una certa notorietà che si sia rifatto a modelli stilistici francesi. Gli esiti decisamente problematici, dànno ulteriormente la conferma che la Francia ha contato per l'intavolazione di motivi che a noi sembravano tutto sommato inconsueti, aiutando il venire alla luce di ipotesi e metodologie impraticate, di universi culturali e morali inediti e impensabili. E ha servito suggestioni formali, piú che modelli. Cosí ad es. un Italo Calvino non è avvicinabile a nessuno scrittore francese contemporaneo, ma la strutturazione dei suoi racconti, i calcoli geometrici, la "clarté" tutta intellettuale delle sue descrizioni - di un percorso quanto di un bicchiere, di un labirinto o di un numero - sarebbero inimmaginabili senza il lavoro teorico e analitico compiuto in precedenza, con i francesi naturalmente in prima linea. Lo stesso romanzo di Umberto Eco, Il nome della rosa, non va esente da uguali influssi, anche se li coniuga con moduli anglosassoni e postmoderni.

È comunque nell'intervolto specificamente critico oltreché nella produzione delle idee e dei linguaggi che le espongono, che s'agitano maggiormente i termini reali dell'affabulazione esercitata sui foresti italici ammalati di francioserie. I quali ultimi (ecco la flessibilità ricordata da Franco Fortini!) sono riusciti cammin facendo a rovesciare cielo e terra e a cambiarsi nell'opposto di tutto, tuffati nel febbrile andirivieni delle idee correnti e ricorrenti, acquisite e innovanti. In luogo di dare la lista kilometrica dei fuggiaschi dal marxismo e dallo strutturalismo alla volta di un sociologismo selvaggio (l'ultima parola d'ordine di questo scorcio di stagione è l'elogio intinto di meraviglie dell'orrido Flashdance!), ci limiteremo a dire di una evoluzione almeno motivata sul piano culturale, anche se opinabile e non esente da risvolti alla moda. Pensiamo a Gianni Scalia.

Dai disegni scultorei del suo ipermarxismo di battaglia, Scalia è passato attraverso l'opera di riletture e ripensamenti contrassegnati dalla rivista Per la critica (redatta nel biennio '73-'74), alle linee nebbiose della fase dell'opposizione (Il cerchio di gesso), via 
via sino alla scoperta di Heidegger e del carattere di specificazione, anche ideologica, del linguaggio poetico nel suo stesso venire alla luce. In forma di parole si intitolano infatti i libri di una nuova edizione periodica (l'ultima in cui Scalia si sia buttato, nevroticamente scavalcando le precedenti impostazioni e intraprese), che ha allineato tra gli altri il Federico Garcia Lorca di Viaggio verso la luna $^{22}$ (una sceneggiatura per film), Maurice Blanchot ${ }^{23}$ e quel grande interprete dell'universo che è Edmond Jabès. ${ }^{24}$

"Siamo convinti," ha osservato Scalia, a nome del gruppo di lavoro, nell'opuscolo informativo destinato ai lettori, "ostinatamente e lietamente, che lo scrivere e il leggere siano ancora insostituibilmente vivi, siano dell'ordine, intimo e pubblico, del frui, non dell'uti finché, almeno, ci siano liberi scrittori e, come si diceva una volta, liberi 'leggitori'."

"Nello spazio e nel tempo della parola, cosí prepotentemente occupato, c'è bisogno di una fessura, di un interstizio, d'una benefica arguta effrazione: una forma, appena udibile e visibile, di separazione; non destinata ai circuiti fallimentarmente alternativi, alla piú o meno rapida estinzione, alla elegante o volgare entropia." 25

Il gioco degli specchi e delle duplicazioni, mentre intellettualmente si è dilatato allargando i suoi propri giri, qui è però giunto a una misura di fisicità tutta italiana, anche attraverso la riscoperta della parola. Res sunt nomina: aveva scritto Pasolini, ${ }^{26}$ uno dei punti di riferimento dell'ultimo Scalia ${ }^{27}$ (ma anche di autori piú o meno giovani che potrebbero essergli, almeno per qualche tratto, affini: pensiamo ad esempio a poeti, come Gianni D'Elia, ${ }^{28}$ Pier Giorgio Paterlini, ${ }^{29}$ Franco Scataglini). ${ }^{30}$

$\mathrm{E}$ infatti, come ha rimarcato Jabès stesso, per "esistere, prima di tutto è necessario essere nominati; ma per entrare nell'universo della scrittura, bisogna avere assunto, con il proprio nome, il destino di ogni suono, di ogni segno che lo perpetua." ${ }^{31}$ Edmond Jabès ha ricevuto il Premio Pasolini 1983. È uno dei non molti casi in cui il colloquio con l'enclave parigina è stato importante e proficuo.

Ma torniamo a Roland Barthes: al Barthes scrittore, sia pure nell'ambito saggistico, che ha infine superato e messo a tacere tutte le interpretazioni rigide del discorso espressivo e connotativo. Non so in quanti si siano avveduti che l'inizio de La chambre claire fa riemergere, dissimulandolo e intanto spostandone il senso, l'inizio 
della Recherche. L'affiorare dell'avverbio di tempo - "longtemps" - è il segnale appariscente e tuttavia meno sintomatico. Il tratteggio cronologico, affidato alla forma verbale, rimarca essenzialmente la scoperta dell'evento, da cui poi arriva il movimento di entrambi i libri: l'immersione nel passato per Proust, le movenze del discorso sull'immaginazione fotografica in Roland Barthes. Invece che l'avverbio, sono appunto il verbo e l'annessa locuzione di tempo ad accelerare il disvolgimento dei due rispettivi nuclei.

Il primo paragrafo della Chambre claire ha una verticale di penetrazione tutta compendiata di verbi narrativi: "Un jour ... je tombai sur une photographie ... Je me dis alors ... Je parlais parfois, "32 e via di seguito. Il percorso dell'analisi si concilia pienamente con la rispondenza dello stato d'animo: anzi, è il profilo di quest'ultimo, riafferrato nel giudizio sul mondo ("la vie . . . faite à coups de petites solitudes"), ${ }^{33}$ che compone l'ordito del discorso. L'intrico dei problemi - tradotti in eventi psicologici e anche esistenziali — va a un certo punto dentro l'inevitabile ingorgo. "Qui pouvait me guider ?"34 si trova ad esclamare Barthes sull'onda del pathos delle questioni drammatizzate.

Lo sguardo sull'oggetto è l'antemurale, la prima delle soglie superate dallo scrittore. Subentra, dopo, all'interesse per i dati culturali il désir, il ritorno a se stesso o al referente (cui, secondo Barthes, ogni immagine sarebbe connaturale). È una distinzione che riappare nella struttura della Chambre claire (la prima parte indirizzata alle foto; la seconda concentrata sullo struggimento per la fine della madre, con l'immagine di lei adolescente nel Jardin d'Hiver che ha la forza di un background memoriale). La dissociazione è la medesima del soggetto, dell'autore: sballottato tra il discorso della critica (attraverso i rigagnoli sociologico e semiologico, biografico come altrettanto psicanalitico) e il linguaggio creativo; aggrovigliate dalla completezza del primo ma adesso incline alla sola scrittura che possa universalizzargli il ricordo. In breve, proiettato verso un linguaggio che rifugge dal surcodificare per definire e delineare in proprio: "Ce que j'avais remarqué au début, d'une façon dégagée, sous couvert de méthode, à savoir que toute photo est en quelque sorte co-naturelle à son référent, je le découvrais de nouveau, à neuf, devrais-je dire, emporté par la vérité de l'image." 35

Ben inteso, Barthes nel suo libro non cessa di rapportarsi all'oggetto investigato, la fotografia per l'appunto (ma altrove si trattava d'altro: semiologia, moda, feticci consumistici, ecc.) e in cosí fare, ripone incessantemente in causa il suo stesso lin- 
guaggio. Il peso dell'oggetto di relazione, ottundendo la spinta della tensione ("l'amour extrême"), ${ }^{36}$ oscura alla fine il ritratto che il critico ci offre a mezzo del suo testo. Tale ritratto non sempre coincide con l'io profondo: il volto angosciante e disinteriorizzato, strambo e slavato - che Barthes ad un certo punto scorge in una sua immagine - è in fondo il riflesso di una decezione, dovuta ai limiti di espressione del discorso critico. Eppure, il tratto che a Roland Barthes appare come quello che piú lo respinge e appiattisce su linee rigide, è lo stesso che permette di cogliere le sue vibrazioni tra vita e pensiero, esistenza e riflessione, evento e linguaggio. Il che, in termini diversi, significa che Barthes è scrittore proprio nella incredibile simultaneità fra tracciato critico e la pulsione, l'inclinazione, verso un qualcos'altro che lo trascenda.

All'indice decisivo dello sguardo che s'allarga sull'immagine, il proposito di Barthes era non tenersi irreggimentato dentro i margini della sua dimensione: "face à certaines photos, je me voulais sauvage, sans culture. ${ }^{\prime 37}$ Ma è unicamente stando in piedi nel suo ipogeo di "grand réthoriqueur" che gli è stato possibile superare lo spazio dell'indagine accademica e dotta. Le antiche categorie della critica, messe a lucido e riverniciate, intervengono per organizzare il nuovo quadro culturale, ma con esso anche il campo espressivo. Pietrificati nelle antiche definizioni e in fondo ignari di quanto s'è venuto modificando, gli scrittori di "fiction" francesi non riescono a produrre un discorso convincente e che faccia presa.

Non cosí i prosatori specialistici: che sanno portare la loro scrittura su diversi ritmi e velocità. Non è solo il caso di Barthes, benché il suo sia l'esempio maggiormente rilevante per freschezza e souplesse. La conoscenza che dissolve i tratti accidentalmente fissati dai D'Ormesson e dagli Ajar - ma anche dalle Cardinal, perché no? - è quella che ci arriva dalle macchine desideranti, dalle cronichette etnografiche lévistraussiane, dalla "séduction" di Baudrillard, o anche dalle riflessioni di Foucault sull'ordine del discorso. Insomma, l'azzurrina intensità che solo patisce l'uomo senziente la troviamo nella prosa dei critici e degli studiosi, non già in quella degli scrittori vuoi di routine vuoi creativi. ${ }^{38}$

In breve, è successo in Francia in modo clamoroso, dopo però che un'identica tendenza si era sviluppata anche se a un livello sempre medio nel mondo culturale anglosassone e nell'area piú ricca dell'Europa centrale, che ai saggisti, ai filosofi, agli storici in genere, è stato accordato senza troppi patemi e riserve lo statuto di autori. In Italia questo non era mai accaduto: la distinzione tra 
ordine critico e ordine creativo era stata sempre rigidamente mantenuta, se mai studiosi di valente e di grossa intelligenza - primi Luigi Russo, Concetto Marchesi, Ranuccio Bianchi Bandinelli, poi negli ultimi anni Magris, Sergio Quinzio, Guido Ceronetti - si erano trovati con pagine marginali, e non scientifiche in senso immediato, sulle mosse d'andare verso una piú marcata inventiva linguistica, senza doverla affermare come fine primario della loro scrittura. L'esempio (appunto desumibile da modelli francesi, primo tra tutti Barthes) era l'argomentare le tesi filosofiche, oppure storiche, oppure morali e politiche, non insistendo sul filo dello specialismo e della routine e invece abbondando in metafore. È in fondo ciò che Giorgio Pasquali, uno dei maestri della filologia italiana, definí a suo tempo "pagine stravaganti": ${ }^{39}$ dove la stravaganza, non soltanto tematica, infine si palesa come rimozione dalla norma stilistica del linguaggio critico, cosí come comunemente veniva praticato e accepito in sede di lettura e ricezione.

Altro ovviamente è il discorso da farsi per le note saggistiche di narratori e poeti: da Fortini a Moravia, da Testori a Volponi, al Pasolini pirata e luterano, al quale si deve l'immagine che piú ha suggestionato la coscienza del pubblico colto e della società civile degli anni '70, quella del Palazzo ${ }^{40}$ (simboleggiante il potere e i suoi evidenti connotati storici, con tanto di anagrafe indirizzo attribuzioni).

È un discorso che si diceva alquanto diverso, perché in quei casi le muricce divisorie tra arte e riflessione, tra inventio e critica del reale e del sapere, venivano meno in modi naturali e immediati. Molto invece $c^{\prime}$ è voluto perché l'eccezionale vivificazione linguistica di un Roberto Longhi ${ }^{41}$ venisse riconosciuta per tale al di là del primato della sua analisi sul corpo delle arti figurative (ma perché si arrivasse a tanto, s'è dovuto scomodare Gianfranco Contini, ${ }^{42}$ con tutto il peso del suo prestigio e del suo stesso potere accademico). Del resto ancora oggi si stenta a dare a Cesare Brandi $^{43}$ - per ritrarsi ancora al quadro della critica figurativa ed estetica - quanto gli spetta, scrivendo egli come fa "en artiste" e "en poète" (quanto alle abbaglianti e mosaicate effulgenze della sua prosa) assai piú e meglio dei tanti che confidano a versi e rime, racconto e fantasia, la volatilità del proprio io.

Ora, non è dei lettori e degli studiosi italiani chi non sappia quale prestigio e quale forza di suggestione avesse acquistato il Barthes interprete del sistema di segni della realtà e della letteratura, della moda e dei simulacri di massa. Tutto ciò, almeno in Italia, ha sortito l'effetto dell'ammirazione e di un rinnovato spi- 
rito critico. Ma non altrettanto si può dire che ne sia discesa una scuola o corrente barthesiana, cosí come invece si è potuto parlare di filoni althusseriani, foucaultiani, lacaniani (la mappa delle accolite freudiane piú o meno ortodosse, di ascendenza francese e non, è invece cosí complicata che intenzionalmente ci si astiene dal riferirne in questa sede).

Nullameno, dopo che nei libri di Barthes il linguaggio verbale ha preso a declinare in quelle flessioni intonative capaci di offrire un alone affettivo diverso alle parole, affermando al contempo sulla pagina piú cose che non ne sarebbero venute dai volumi di critica e di analisi letteraria, dato ovviamente il carattere polisemico del linguaggio che designa al di là della pura e semplice comunicazione, i tassi di filtrazione e di impollinazione sono aumentati. Almeno nel senso che anche in Italia molti si sono concessi nella scrittura critica, e se lo sono visti riconoscere, una assai piú ampia latitudine di procedimenti e intuizioni.

In forza del valore d'esempio del modello barthesiano (ma dopo anche la riabilitazione della metafora fatta da Lacoeur Derrida Lacan Jakobson Perelmann), anche da noi la figura del saggista ha ottenuto finalmente il suo statuto di autonomia (Pasolini disse, nei suoi ultimi articoli stampati in Tempo settimanale, "descrizioni di descrizioni"), ${ }^{44}$ trovandosi slegata dalle posizioni e ingessature tradizionali.

Come sia potuto accadere, o quale fondazione teoretica tutto questo abbia, è ancora una volta esplicato in casa francese. Ad esempio: trattando in Dialogues ${ }^{45}$ dell'inabissamento della psicanalisi, Claire Parnet (che, lo si ricorderà, intreccia una conversazione con Gilles Deleuze) sostiene che al posto delle funzioni hanno corso nell'opera funzionamenti macchinici con concatenamenti collettivi. L'esempio piú persuasivo è per l'appunto Roland Barthes: che dal concetto di "significante" sopravviene a un suo regime - suo e insieme collettivo - di particolare "passionalità." Sotto il plafond contornato da lessico e linee strutturali, Barthes rintraccia uno spazio che è invece mulinante di segni: su cui interviene - come fa ad es. con Sade e Charles Fourier, ma soprattutto con Ignacio de Loyola - colorandone i riquadri e le bordure. La presenza, dietro le luci dell'apparenza, di ogni flusso coniugato e di ogni pluralità di divenire, spiazza quanti insistano su finzione e espressività personale. Il libro diviene ascesi spirituale, o un regime di segni con cui si congiungono al tempo stesso flussi d'espressione e flussi di contenuto, determinando su di questi la presenza di concatenamenti di desiderio e sui primi quella dei concatena- 
menti di enunciazione, a loro volta gli uni presi dentro gli altri. Nel primo caso, il richiamo può andare ai Fragments d'un discours amoureux, che in Italia ha trovato un corrispettivo degradato e banale in Innamoramento e amore del sociologo Francesco Alberoni; nel secondo, si può pensare a La chambre claire.

L'esito conclusivamente toccato da questa tendenza è che mai come in questi ultimi anni si sono letti in Italia saggisti e teorici, filosofi e storici; ma mai s'è registrata una indifferenza cosí generale e diffusa per la letteratura francese contemporanea, quella dei poeti e dei romanzieri. Il perché - ancora una volta - è meno da cercare da noi e piú invece da individuare nelle cose parigine (il che torna a conferma che sotto questo aspetto l'intellighenzia italiana subisce molto il trambusto delle mode e delle retoriche, volta a volta gabellate per realismo impegno psicanalisi post moderno spazzatura).

Torniamo dunque a bomba: per dirla con un linguaggio colto e affilato, letterariamente in sintonia col discorso che qui si traccia, "à nos moutons." Sempre Barthes ci ricorda il principio d'avventura. ${ }^{46} \mathrm{Si}$ prenda un oggetto o un quadro, un corpo oppure un paesaggio (nel caso menzionato ne La chambre claire è ovviamente questione di immagini fotografiche). C'è qualcosa che raggiunge il dominio dell'interesse; qualcos'altro che, invece, smargina da ogni forma d'accettazione serena e consapevole, per toccare l'emozione e il dominio interiore. È ciò che Barthes denuncia di fronte a certe immagini, quelle e non altre; ma che potremmo stendere a un corpus letterario. Ora, se torniamo all'ipotesi di Deleuze e Parnet sui sistemi di collegamento (non il punto ma le linee, non un soggetto quanto il divenire insieme), che cosa, nel teatro quotidiano di attività rigorosamente prestabilite, impedisce agli scrittori francesi di incontrare in un tratto quella nostalgia e quell'inquietudine che è "pression de l'indicible qui veut se dire ${ }^{\prime \prime} ?{ }^{47}$

Il principio d'avventura richiamato da Barthes serve ad indagare il flusso inatteso che arriva dall'oggetto, animandoci ma allo stesso tempo spingendo ad animarlo a nostra volta. La distinzione tra i due linguaggi si replica allora nel diverso atteggiamento davanti all'oggetto relazionale: da un lato un approccio mosso da un interesse eminentemente culturale (Barthes lo designa attraverso il latino studium $){ }^{48}$ dall'altro la ferita che, partendo dal testo, raggiunge e spinge, sconnette e disperde. Il lemma latino punctum: ${ }^{49}$ l'espansione del quale non è caso intentivamente proustiana.

Addensare giunture disvolgendo situazioni e modi ricevuti dall'esterno è dunque possibile alla condizione di lasciarsi incontami- 
nabile a un riguardo culturale: scivolando su linee di pendenza con sussidio del sentimento. Nel linguaggio di Deleuze e della Parnet, questo significa una forma di distacco dal reiterarsi del noto. Tanta propensione da un lato, quanta indipendenza da ogni idée reçue dall'altro. L'impotenza del romanzo francese di questi anni, per colmo di contraddizione, è cosí immane alla complessità delle sue strutture. A quel suo trovarsi su linee cervellotiche e neutre, facendo astrazione da ogni principio di piacere o piacere della lettura. Già questo spiega la disinformazione degli italiani, che pure sono tornati, dopo il '68, alla letteratura creativa.

Se questa è la situazione, la ricetta di Dialogues è distruggere il codice-territorio acquisito: diventare transfughi dal proprio universo ed evadere per cosí penetrare in altre vite: affacciandosi alla soglia di congiunzione dei diversi e sfuggevoli movimenti su linee che siano al contrario di fuga. Ma non già fuga dal mondo e dal reale, dai gesti umani e dalle responsabilità. Fuggire, osserva in proposito Deleuze, significa tracciare una linea, delle linee, tutta una cartografia. Si scoprono dei mondi solo in una lunga fuga spezzata. In tale "deterritorializzazione" consiste - o consisterebbe (personalmente non ne sono sicuro) - la superiorità della letteratura anglo-americana. Ma il gusto dei lettori italiani, per ciò che esso vale, combacia con tale linea.

Per contrappeso, si veda invece quella tradizione husserlianoheideggeriana, che trapiantatasi in Francia e alimentata negli ultimi decenni da Lévinas, Beaufret, Dufrenne, si è vivacemente contrapposta al "pensiero calcolante," forte negli Usa e in Gran Bretagna. Dopo Jacques Lacan e Derrida, è stato Paul Ricoeur ${ }^{50}$ a ridisegnare la solidarietà tra lingua rappresentativa e Vorhandenheit: che è un ritorno del linguaggio e del segnico.

Tutto e il contrario di tutto? Forse sí. Ma, piú probabilmente, la lingua rappresentativa non era mai stata lasciata cadere. Soltanto che essendo essa avvicinamento al senso ultimo delle cose, all'Essere dell'ente, cioè all'Essere solo in quanto Essere, non sembrava piú consentito avallare nessuna Vertretung, nessuna forma di rappresentazione sentita oppure gestita tradizionalmente.

Nel lavoro dei francesi, a parte gli eccessi, corrono ovviamente una logica e un metodo. Ora, cosa resta di tutto ciò nel passaggio italiano (insieme con la puntuale riproduzione e gli approfondimenti che pure non sono mancati)? Forse non molto. In ogni caso è ancora presto per dirlo. Ed è anche difficile individuare aree o linee di ricerca nettamente caratterizzate, o ancor piú nettamente 
influenzate. Nullameno, per quanto abbreviativamente, qualche dato piú solido può essere fornito.

Il gruppo maggiormente agguerrito, e piú liberamente ancorato alla nuova cultura francese, ermeneutica ed epistemologica, è forse quello raccolto attorno alla rivista Aut aut (diretta tra gli altri da Pier Aldo Rovatti e Giampiero Comolli, e avvalsasi di collaboratori come Agamben, Baudrillard, Bodei, Cacciari, Deleuze, la Heller, Vattimo). La nuova serie della rivista, il piú sovente cucita su numeri monografici, ha indagato i legami tra potere e saperi (in un fascicolo, il 167-68 del 1978, che era stato concepito come numero speciale dedicato al pensiero foucaultiano), il lascito di Lacan (n. 177-78, maggio-agosto 1980), la ricerca storica (n. 181, gennaio-febbraio 1981), le implicazioni filosofiche della Recherche (n. 193-94, gennaio-aprile 1983). Due fascicoli speciali di Nuova corrente sono stati invece consacrati a Gaston Bachelard e la scienza (n. 64 del '74), e dopo, nel 1981, a Derrida o la lezione del calcolo (n. 84). Ma è tutto il lavoro di queste riviste ad essere impastato di spirito francese. Senza comunque generalizzare, si può sostenere che sono le aree piú inquiete e problematiche, piú latamente asociali, a rivolgersi il piú frequentemente alla cultura e alle impostazioni d'oltralpe. Diversamente, è il mondo anglosassone a dare incentivi e argomenti a settori culturali un tempo influenzati dal marxismo, e oggi invece attenti a analisi lontane da ogni ideologizzazione del reale (vedi l'équipe di Lavoro politico, con Alberto Asor Rosa, Mario Tronti, Giacomo Marammao, Gian Enrico Rusconi, Massimo Cacciari, Remo Bodei).

Per infine conchiudere: escludendo ogni influsso di scrittura narrativa e poetica in senso tradizionale (lo stesso Philippe Sollers, sponsorizzato dalla rivista Spirali in un convegno a lui appositamente dedicato, ${ }^{51}$ non ha trovato né seguaci e ripetitori del suo stile, né interpretazioni e rielaborazioni creative) e dopo avere indiziato la saggistica e l'ermeneutica come il terreno di reale confluenza delle energie piú attive, diremmo irrefragabile che gli intellettuali italiani abbiano sarchiato dalla griglia francese ciò che potremmo dire il 'principio dell'incertezza,' esteso a tutte le sfere, dalla letteratura alla filosofia, dalla sessualità alla psiche al politico.

Però di tale principio non sono stati recepiti i risvolti critici, e soprattutto le chiavi che avrebbero aperto l'accesso a una dimensione visionaria e lucida, come conoscenza e pratica di ciò che non è neppure nominabile (che resta in fondo il contributo piú importante della cultura francese degli ultimi vent'anni). Céline e 
Genet, Drieu o Sade, pur letti e anche osannati, non hanno attecchito nel terreno italiano. Il solo esempio di un uso conseguente della trasgressione sadiana è forse quello di Salò o le 120 giornate di Sodoma, ${ }^{52}$ il film postumo di Pier Paolo Pasolini. Ma lo stesso grande poeta de Le ceneri di Gramsci ci è tornato dai convegni e dalle letture francesi sempre svuotato di quella carica outrée e radicalmente alternativa che i saggisti e gli autori parigini gli hanno riconosciuto. ${ }^{53}$ In tutto ciò il positivo è solo il rifiuto delle riduzioni e identificazioni edonistiche, tipo il Fernandez di Dans la main de l'ange.

Ma in particolare, sono state esasperate certe letture e certe impostazioni, che piú nettamente avevano rotto con il passato. Accenniamo sia pure di sfuggita a Lyotard, ${ }^{54}$ molto citato ma sostanzialmente non capito: infatti il post-moderno in Italia è stato ed è repertazione archeologica e retorica. Forse è importante ricordare il concetto di "séduction" di Jean Baudrillard, ${ }^{55}$ accepito meccanicamente per dare impulso e giustificazione ai linguaggi piú spenti e mercificati (linea perseguita nell'ambito della ricerca cinematografica da parte soprattutto dei cosiddetti giovani filologi, ${ }^{56}$ che non sono tali proprio per nulla); ed è da dire della nuova scuola storica, che ha in Carlo Ginzburg (Il formaggio e $i$ vermi, Indagine su Piero) la sua punta di diamante: scuola rilegantesi alle Annales e a Fernand Braudel, ma del tutto scalcata da quel modulo dialettico che nell'opera del maestro francese era appunto in grado di saldare le analisi della 'civilisation matérielle' sullo zoccolo di letture precedenti, quella ad esempio di Marx, in tal modo arricchendole. Ha osservato infatti Braudel: "è importante affrontare tutti i nostri problemi (tanto diversi da quelli che lui ha conosciuto) nello spirito che era, o che mi sembra, il suo. ${ }^{57}$ Non altrettanto può dirsi il metodo di certi suoi nipotini italiani. Ma quella di Karl Marx è una nota dolente: la registrazione banale di una banale identificazione con il gulag (sulla scorta delle allegre facezie di Levy Dollé Benoist Clavell Glucksmann e ruminanti affini) e con la liquidazione della ragione dialettica. Gli ex-filocinesi di Tel Quel sono divenuti in un colpo solo metafisici e mistici? Anche in Italia si riscopre lo spirito, la conservazione, l'antimateria. Ecco allora Maria Antonietta Maciocchi, che prima aveva spiegato dai pulpiti di Nanterre il revisionismo del Pci usando un Gramsci miscelato a Mao, saltabeccare tra i fossati del post-marxismo. Ecco Calvino dichiararsi deluso della storia; ecco gli ex-contestatori trasformarsi d'incanto in monaci buddisti. Eppure Althusser ammoniva che in Marx c'erano domande, cui serviva trovare una risposta adeguata: domande 
sull'alienazione, il feticismo, il concetto di catastrofe, quello epistemologico. Purtroppo, il semilavorato del filosofo francese non ha incontrato approfondimenti e sviluppi apprezzabili, e meno che meno da noi, dove il modello di partenza è in sostanza sempre immeschinito.

Per finire: anche nel rapporto con tutte le ipotesi, le ricerche, le posizioni dei nostri cugini d'oltralpe, il principio dell'imitazione e della citazione passiva pare essere in Italia la moneta corrente. Pure qualche volta esso diviene personale: si fa mimesis praxeos, che è anche senso e produce senso nella comprensione dei modelli cui si riferisce in piena autonomia. Non accade spesso. Ma quando accade, la cultura italiana recupera la propria peculiare vocazione e centralità.

\section{Università di Urbino}

\section{NOTE}

1 L'articolo che dà fuoco alle polveri è di Giovanni Giudici ("Semiologo, ti dichiariamo guerra," in L'Unità, 22 febbraio 1983); ad esso immediatamente replica Omar Calabrese ("Non nascondetevi dietro Paperino," in L'Unità, 26 febbraio 1983), ma in precedenza c'era stata la pubblicazione in rivista di un importante saggio di Roger Shattuk, "Come salvare la letteratura," in Comunità, 184, XXXVI (ottobre 1982), e soprattutto un libro di Alfonso Berardinelli, Il critico senza mestiere (Milano: Il Saggiatore, 1983), in cui l'ultimo testo, che porta il medesimo titolo, fa un esame sconsolato della situazione italiana: "Da un lato (nell'accademia) la critica letteraria non desidera altro che essere il degno e impeccabile prolungamento di qualcuna delle piú accreditate scienze umane (linguistica, psicanalisi, sociologia). E dall'altro (nel giornalismo) la critica tira avanti come può al servizio delle circostanze, senza metodo e senza criterio, incapace di mettere in gioco un'idea o un progetto, dichiarando con fierezza un disprezzo totale per i vecchi giudizi di valore e insieme (causa di forza maggiore) non facendo quasi altro che pronunciare una catena ininterrotta di giudizi di valore: per lo piú infondati e a proposito di opere che attraversano il mercato librario come saette" (pp. 125-26).

Come si vede, non sono argomenti e accuse di scarso rilievo. Dopo d'allora, il contenzioso si è allargato alle pagine de La Repubblica, Il Manifesto, Il Messaggero, Panorama, Quaderni Piacentini.

2 "I metodi semiotici formulati in America, in Francia, in Italia, in Russia, hanno incominciato a confrontarsi e a convergere, dando vita a una critica letteraria che ha rivoluzionato l'interpretazione dei tempi. Anzi le sole cose originali e solide prodotte dalla critica dell'ultimo ventennio appartengono proprio all'area della semiotica o alla sua periferia": Cesare Segre, "Quei nostalgici del fanciullino," in Tutto libri, anno IX, n. 353, supplemento a La Stampa del 2 aprile 1983, 1.

3 Alfonso Berardinelli, "Tipi intellettuali: ruspa, tritacarne, apriscatole, frullatore," in Linea d'ombra, 3 (ottobre 1983), 57.

4 Cfr. Maria Corti, "Una pseudo-querelle," in Alfabeta, anno 5, numero 50-51 (luglio-agosto 1983), 4.

5 Franco Brioschi, "Il convitato di pietra," in Linea d'ombra, 3 (ottobre 1983), 66. 
6 "Con la propria modesta metafora quel titolo non allude insomma a nessuna superiorità di vedute né ad alcun conflitto guerreggiato. Finta modestia, va da sé. Allude ad aree infradisciplinari, a punti di contatto o di frizione fra conoscenze, intenzioni, finalità diverse. Allude ai temi intorno ai quali ruota la maggior parte di questi miei scritti giornalistici; e che a distanza di tempo mostrano, perché non dirlo?, una certa unità o ostinazione. È l'area dove vorrei fosse impossibile distinguere fra giudizi letterari, considerazioni di costume, critica della cultura, valutazioni politiche": Franco Fortini, "Quando ci si muove in zona contestata," in Corriere della Sera (11 gennaio 1984), 3.

7 Scrive Fortini nello stesso articolo: "A me, che non so se sono mai stato un critico marxista e che di quei complessi non mi pare di aver sofferto, l'osservazione pare esatta. [Si richiama a un rilievo di Brioschi, nell'intervento sopra ricordato, relativo a un senso di colpa della critica marxista e di sinistra.] La flessibilità (questo è un eufemismo) intellettuale che nell'ultimo decennio ha affollato di marxisti pentiti le anticamere dei semiologi non mi stupisce davvero" (lbid.).

8 L'espressione è usata dal Manzoni per indicare le miserabili transazioni e insipienze dei medici di fronte alla peste: è il capitolo XXXI dei Promessi Sposi (Firenze: La Nuova Italia, 1950), p. 590.

9 Si tratta di Che cosa è lo strutturalismo di Gianni Puglisi (Roma: AstrolabioUbaldini, 1970).

10 Roland Barthes, Critica e verità (Torino: Einaudi, 1969), p. 63 (l'edizione francese venne impressa nel '66).

11 Cfr. Louis Althusser, Pour Marx (Paris: Maspero, 1965).

12 Cfr. Eleonora Fiorani, Friedrich Engels e il materialismo dialettico (Milano: Feltrinelli, 1971), e poi ancora Eleonora Fiorani, Ferdinando Vidoni, Il giovane Engels. Cultura, classe e materialismo dialettico (Milano: Mazzotta, 1974).

13 Si veda Félix Guattari, La révolution molécolaire (Fontenay-sous-Bois: Editions Recherches, 1977), con particolare riferimento ai primi tre articoli.

14 Cfr. Michel Foucault, Microfisica del potere (Torino: Einaudi, 1977). L'edizione italiana è arricchita da un'intervista dell'autore ai due curatori, Allessandro Fontana e Pasquale Pasquino.

15 Cfr. Alberto Asor Rosa, "C'è anche una fisica del potere," in L'Unità (26 ottobre 1977), 3.

16 Sulla filiazione del pensiero dalla "convenance" del discorso, dunque sui rapporti della "nuova filosofia" con la semiotica parigina, si può leggere con profitto "Discorso e realtà" di Luis J. Prieto, in L'Unità (4 ottobre 1977), 3.

17 I nuclei teorici al centro de La teoria dei bisogni in Marx (che Feltrinelli pubblicò nel '74 con una prefazione di Pier Aldo Rovatti) divennero le ragioni critiche del cosiddetto Movimento del '77.

18 L'articolo delle lucciole, comparso nel Corriere della Sera del 10 febbraio 1975 col titolo "Il vuoto del potere in Italia," è stato inserito da Pasolini stesso nei suoi Scritti corsari (Milano: Garzanti, 1975), pp. 160-68.

19 Leonardo Sciascia, L'affare Moro (Palermo: Sellerio, 1978), p. 126.

20 Molte sono le opere di Antonio Negri, edite da Feltrinelli, tutte ugualmente inficiate da un estetismo rivoluzionario a malgrado del rilievo teoretico: ricordiamo tra le altre Il dominio e il sabotaggio (1978), Marx oltre Marx (1979), Crisi dello Stato-piano (1979), Il comunismo e la guerra (1980), Proletari e Stato (1980), La forma stato. Per la critica dell'economia politica della Costituzione (1980), L'anomalia selvaggia (1982).

21 Leonardo Sciascia, Nota dell'autore a Candido (Torino: Einaudi, 1977), p. 139.

22 Cfr. Federico Garcia Lorca, Viaggio verso la luna, introduzione di Marie Laffranque, commenti di Antonio Melis, Paola Micheli, Maria Grazia Profeti, con una scelta di disegni dell'Autore (Reggio Emilia: Eliotropia Edizioni). 
23 Cfr. Maurice Blanchot, La follia del giorno. La letteratura e il diritto alla morte, con letture di Jacques Derrida e Emmanuel Lévinas (Reggio Emilia: Eliotropia Edizioni).

24 A Enrico Filippini che gli chiedeva se l'ebreo privo di terra trovasse la propria patria nel libro, Jabès ha risposto che era proprio cosí: "Il libro, la Torah, va letto e commentato all'infinito. La sua verità è dietro ciò che si legge, e non è mai fissata: la pratica dell' ebraismo è uguale a quella dello scrittore. È questa scoperta, se posso chiamarla cosí, che mi ha avvicinato a quello che io chiamo un ebraismo ateo, proiettato in un'apertura indefinita. . . . La domanda, I'interrogazione è l'apertura . . ." (La Repubblica, 5 maggio 1983).

25 Gianni Scalia, Ai lettori in I Libri di "In forma di parole," opuscolo propagandistico (Reggio Emilia: Eliotropia Edizioni, 1982), p. 3.

26 "Ho da tempo l'ambizione di scrivere una 'Filosofia' del cinema, consistente nel rovesciamento del nominalismo: non nomina sunt res ma res sunt nomina. Se $c^{\prime}$ è una decifrazione della realtà ci deve essere a fortiori una cifrazione; se c'è un decifratore, un cifratore" (cosí Pasolini in un saggio per Bianco e nero, del marzo-aprile 1971, poi ripreso in Empirismo eretico [Milano: Garzanti, 1972], p. 261).

27 Ha scritto Gianni Scalia: "Pasolini è . . . una voce e una persona che non si sostituisce: che ci manca ogni giorno di piú. O io almeno lo credo: la 'richiesta di poesia,' il 'sogno di una cosa,' la 'mania della verità' (se provassimo a pensarle, queste verità, ad una ad una, e metterle insieme?) sono necessarie. Sono anche state, nel linguaggio e nel corpo di Pasolini, le verità di un 'comunista comune,' di un 'marxista ordinario,' di un 'compagno, non compagno.' $O$, meglio, di qualcuno che è, forse, già al di là: che insegna a non dimenticare la crudeltà necessaria per la trasformazione di noi e delle cose e che 'non c'è crudeltà senza una specie di coscienza applicata' (Artaud)": Introduzione a La mania della verità (Bologna: Cappelli, 1978), p. 9.

28 Gianni D'Elia, autore di una raccolta poetica dal titolo di Non per chi va (Roma: Savelli, 1980), si è insistentemente richiamato alla figura di Pasolini. "Nessuna poesia piú di quella di Pasolini, in questo secolo, porta dentro di sé l'istanza di questa ragione vitale, l'evento ossesso del corpo. Eे questa istanza evenemenziale ciò di cui fa esperienza il linguaggio pasoliniano": "L'amore della lingua," in Lengua, numero zero (febbraio 1982), 45.

29 Pier Giorgio Paterlini è un giovane poeta emiliano dalla vena fresca e immediata, un po' a mezza strada tra Pasolini e quel grande poeta che è Penna. $\mathrm{Ha}$ pubblicato I salici inutili (Forlí: Forum, 1977) e 11 tempo totem (Forlí; Forum, 1982).

30 La convergenza di Franco Scataglini con la lirica pasoliniana è da rilegare alla querelle sulle culture locali e sull'uso del dialetto. Scataglini è infatti un poeta che si serve del suo gergo materno, quello anconetano, però lavorandolo a modo di lingua. Ha pubblicato: Echi (Ancona: Ata, 1950), E per un frutto piace tutto un orto (Ancona: L'Astrogallo, 1973), So' rimaso la spina (Ancona: L'Astrogallo, 1977), Occorrimenti (Sora-Padova: Edizioni dei Dioscuri, 1980), Carta Laniena (Ancona: Residenza, 1982).

31 Cfr. Opuscolo in Il libro delle interrogazioni (Reggio Emilia: Eliotropia Edizioni, 1982), p. 9.

32 Roland Barthes, La chambre claire (Paris: Cahiers du Cinéma Gallimard Seuil, 1980), p. 13.

33 ld.

34 Ibid., p. 14

35 Ibid., p. 119

36 Ibid., p. 27.

37 lbid., p. 20.

$38 \mathrm{Ha}$ osservato al riguardo Fernand Braudel: "se la Francia non ha piú quella letteratura di cui si gloriava, se ripiega sui suoi filosofi e sulle sue scienze sociali, 
su una fanteria mal preparata al combattimento, è forse perché, contrariamente a quanto si pretende, la Francia non ha sofferto abbastanza? La Francia di JeanPaul Sartre non è la Francia di Balzac. Che la Francia soffra, dunque: la sua letteratura riprenderebbe splendore. Come vedete, un francese non ha uguali quando si tratta di dire male della Francia": "I diletti romanzi che non leggo piú," in Corriere della Sera (13 dicembre 1983), 3.

39 Come si sa, le Pagine stravaganti di un filologo apparvero a Lanciano da Carabba nel 1933; seguirono, nel ' 35 e nel ' 42 da Sansoni, le Pagine meno stravaganti e le Terze pagine stravaganti; poi, nel '51, da Neri Pozza, le Stravaganze quarte e supreme, e nel '52, per l'editore De Silva di Firenze, le Pagine stravaganti vecchie e nuove: il tutto ripresentato dalla Sansoni nel '68 con il titolo Pagine stravaganti (due volumi).

40 Cfr. Pier Paolo Pasolini Lettere luterane (Torino: Einaudi, 1976), pp. $92-98$ e passim.

41 Rimandiamo alle bellissime pagine raccolte in $\mathrm{Da}$ Cimabue a Morandi, a cura di Gianfranco Contini (Milano: Mondadori, 1983).

42 Alla straordinaria perizia e intelligenza tecnico-critica, Contini ha avvicinato "le doti di uno scrittore da annoverare tra i maggiori del secolo in Italia: i sistemi di metafore, adibiti da un'euristica fertilissima ... si apparentano ... pur trascendendole, a quelle equivalenze fantastiche dell'opera poetica che si ritrovano in Emilio Cecchi": Gianfranco Contini, Schedario di scrittori italiani moderni e contemporanei (Firenze: Sansoni, 1978), p. 113.

$43 \mathrm{Su}$ Brandi scrittore, ci si consenta di rinviare ad una nostra scheda critica sul suo Disegno della pittura italiana in Rassegna Lucchese, n. 12 (estate 1982), 43.

44 La rubrica letteraria venne tenuta da Pasolini dal 26 novembre 1972 al 24 gennaio 1975 (Pasolini la interruppe per girare Salò). Nell'ultimo articolo, dedicato a Todo modo di Sciascia, egli scrisse: "Ho fatto delle 'descrizioni.' Ecco tutto quello che so della mia critica in quanto critica. E 'descrizioni' di che cosa? Di altre 'descrizioni,' che altro i libri non sono. L'antropologia l'insegna: c'è il 'dròmenon,' il fatto, la cosa occorsa, il mito, e il 'legòmenon,' la sua descrizione parlata": Descrizioni di descrizioni (Torino: Einaudi, 1979), p. 457.

45 Cfr. Gilles Deleuze e Claire Parnet, Dialogues (Paris: Flammarion, 1977).

46 "Aussi, il me semblait que le mot le plus juste pour désigner (provisoirement) l'attrait que certaines photos exercent sur moi, c'était celui d'aventure. Telle photo m'advient, telle autre non": Roland Barthes, La chambre claire, ed. cit., p. 38.

47 Ibid., p. 37.

48 lbid., p. 77.

49 Ibid., pp. 73-74, sgg

50 Si pensi ad alcune tesi di Temps et récit (Paris: aux Editions du Seuil, 1982), piú altre posizioni, tra l'altro esposte da Paul Ricoeur all'inizio del 1984 all'Istituto italiano per gli studi filosofici di Napoli, che troveranno presumibilmente posto in Temps et récit 2.

51 Per la verità, il convegno (tenutosi a Milano il 27 e 28 marzo 1982) traeva l'occasione dalla presentazione in italiano di Paradis (per i tipi di Spirali): nullameno il suo titolo e il suo argomento erano Il romanzo alla vigilia del terzo millennio.

52 I titoli di testa del film riportano infatti la seguente bibliografia: Roland Barthes, Sade, Fourier, Loyola; Simone de Beauvoir, Faut-il brûler Sade?; Maurice Blanchot, Lautréamont et Sade; Philippe Sollers, L'écriture et l'experience des limites; Pierre Klossowski, Sade mon prochain e Le philosophe scélérat.

53 Ricorderei tra tutti il seminario svoltosi a Parigi nel maggio '79 e oggi raccolto in Pasolini (Paris: Grasset, 1980), e ancora Pasolini ou le mythe de la barbarie di Fabien S. Gérard (Bruxelles: Editions de l'Université, 1981).

54 Cfr. Jean-François Lyotard, La condition postmoderne (Paris: Les Editions de Minuit, 1979): al postmoderno e alle sue implicazioni ha dedicato molta attenzione il periodico milanese Alfabeta. 
55 Le tesi esposte in De la séduction (Paris: Galilée, 1979), peraltro non piú sostenute dall'ultimo Baudrillard, hanno trovato in Italia pedissequi ripetitori.

56 L'espressione è del saggista cinematografico Lino Micciché: con essa si designa l'area della giovane critica cinematografica, di ascendenze cinefile, passata dall'iperideologismo alla semiotica al sociologismo selvaggio, del tutto eccettuata da ogni giudizio di valore estetico non piattamente identificato con l'industria e le nuove tecnologie.

57 Fernand Braudel, "Ringiovanire Marx non è sconfessarlo," in Corriere della Sera (8 gennaio 1984), 3. 